\title{
Comparison of fixed and non-fixed methods of hand-held dynamometry
}

\author{
BALBino NEPOMUCENO ${ }^{1}$, Manoela Porto dos Santos Menezes ${ }^{1}$, Kamilla Ribeiro ${ }^{1}$, and \\ Mansueto Gomes Neto ${ }^{1}$ \\ ${ }^{1}$ Universidade Federal da Bahia
}

May 5, 2020

\begin{abstract}
Introduction: Hand-held dynamometry (HHD) is a technique used for evaluating muscle strength. The reproducibility and reliability of this instrument have been little reported. Method: Healthy individuals aged $>18$ years were recruited. Isometric strength was tested for ten upper limb muscle groups. The fixed method was performed using a system with suction cups connected to the dynamometer through an inelastic belt. However, during the non-fixed method, the examiner supported the device with the hand. The reproducibility and reliability were calculated using the intraclass correlation coefficient (ICC). Result: A total of 25 right-handed volunteers participated. The reliability ICC values (0.89-0.99) of the non-fixed method were higher than those of the fixed method (0.43-0.85). The reproducibility of the non-fixed method was also superior to that of the fixed method. Discussion: The comparison between the HHD methods suggested the non-fixed method has greater reproducibility and reliability.
\end{abstract}

\section{INTRODUCTION:}

Hand-held dynamometry (HHD) is a technique for evaluating muscle strength, conducted through a sustained maximum isometric contraction. These dynamometers are small and less in weight for easy handling and applicability ${ }^{1-5}$. Such characteristics make the use of this instrument viable in the most diverse environments and populations ${ }^{6,7}$. However, there is still little research investigating the psychometric properties of the evaluation method adapted for this instrument in measuring upper limb muscle $\operatorname{strength}^{8-12}$.

The aim of this research was to compare the reproducibility and reliability results of muscle strength evaluation with a hand-held dynamometer using the fixed and non-fixed method.

\section{METHOD:}

The reliability and reproducibility of two HHD methods for evaluating upper limb muscle strength was assessed and compared using a prospective, cross-sectional study.

Participants:

Healthy individuals were recruited by direct call, text message or social network. Participants (male or female) older than 18 years and who signed the informed consent form were included in this study. Exclusion criteria included acute bone, muscle or joint diseases, reduced functional range of motion $(\mathrm{ROM})^{13}$, presenting severe heart disease or neuromuscular diseases, or cognitive limitations that reduced the understanding of motor commands during the evaluation.

Randomization:

The order of the examiner and evaluation method was randomized for all patients with the use of a draw, which was carried out using a brown envelope. 


\section{Instruments:}

Muscle strength was evaluated using a previously calibrated digital isometric dynamometer, (model 01165, Lafayette Instrument Company, Sagamore, USA). A goniometer (ISP, Sao Paulo, BR) was used to properly mark the articular position of the segments for each movement evaluated ${ }^{14}$. All patients had the proximal segment (torso, arm, or forearm) stabilized with the use of an inelastic belt, in order to nullify the effect of synergistic muscle chains.

Procedures:

The examiners were trained according to the evaluation protocol prior to testing. The protocol tests the isometric contraction for ten dominant upper limb muscle groups (shoulder flexors and extensors, shoulder internal rotators and external rotators, shoulder adductors and abductors, elbow flexors and extensors, wrist flexors and extensors). Prior to the measurement, the volunteers were instructed and trained how to perform each of the movements, and a muscle warm-up was also performed for each movement.

The fixed method used a system of suction cups adhered to rigid surfaces that were connected to the dynamometer through a Mulligan inelastic belt ${ }^{15,16}$. During the non-fixed method, the examiner supported the device with one hand, in a direction contrary to the movement, stabilizing the segment proximal to the moving joint under assessment ${ }^{14,17-20}$.

For both evaluation methods, the isometric contraction was sustained for 3 seconds. Each movement was repeated thrice; the largest of the three values was used. The dynamometer was placed in the distal region of the forearm, $5 \mathrm{~cm}$ from the radial styloid process ${ }^{21}$. A muscle recovery time of 90 seconds between tests was ensured for all measurements ${ }^{14}$. If there was visible compensation of synergistic muscles in any of the movements, the volunteer would be instructed on the correct movement, and the measurement would be repeated. The positions adopted for the measurements were based on previous studies $(\text { Table } 1)^{14,17-21}$.

A minimum resting time of 30 minutes was given between the evaluation of each examiner ${ }^{22}$.

Variables of interest:

The concept of reliability is related to the capacity of the instrument and the evaluation method to generate similar results, even when used by different examiners. Thus, reliability was obtained by comparing the highest peak torque of the evaluations for two independent examiners (or inter-examiner reliability). On the other hand, the reproducibility of the evaluation method (or intra-examiner reliability) was obtained by analyzing the similarity between the HHD test and retest by the same examiner.

Statistical method:

The data were tabulated and analyzed using SPSS (version 21.0, Statistical Package for the Social Sciences, Chicago, USA). The qualitative variables were expressed in absolute and relative frequency, while the quantitative variables were expressed as mean, median, standard deviation (SD) and 95\% confidence interval (95\% CI). Analysis of variance (ANOVA) was used to evaluate the different the peak torque means obtained from the two methods. The level of significance level was set at $\mathrm{p}<0.05$.

Prior to recruitment, a sample size calculation based on the results of a previous study ${ }^{21}$ determined 25 participants were sufficient to detect a $10 \%$ variation between measurements and a $3 \%$ SD with $80 \%$ power with $\alpha$ at 0.05 . As this was a cross-sectional study sample loss was not expected.

Reproducibility and reliability of the tests were calculated using the intraclass correlation coefficient (ICC) and categorized using the classifications proposed by Weir $(2005)^{23}$ : "almost perfect" for values from 1.0 to 0.81 , "very good" from 0.80 to 0.61 , "good" from 0.60 to 0.41 , "fair" from 0.40 to 0.21 , and "low" from 0.20 to 0.00 . A Bland-Altman plot was used for the visualization of the agreement between the quantitative measurements obtained in the tests.

\section{RESULTS:}


The sample consisted of 25 volunteers ( $60 \%$ female), with a mean (SD) age of 33.1 (13.4) years, body mass of $72.6(18.3) \mathrm{kg}$ and a height of $1.7(0.1) \mathrm{m}$, all of which were right-handed (Table 2). Similar peak torque values were observed between the two methods, whereby the non-fixed method had with slightly greater peak torque values in relation to the fixed method ( Table 3). However, no statistical difference was found for these differences between the mean torques for any of the movements evaluated.

The reproducibility of the test and retest for the non-fixed method was categorized as almost perfect for all ten movements evaluated (Table 4). The Bland-Altman plot demonstrated a greater degree of agreement between the test and retest of the non-fixed method than that of the fixed method (Figure 1). The reliability followed a similar behavior, with higher inter-examiner ICC values for the non-fixed method when compared to the fixed method (Table 5). The only exception was the measurement of elbow extension where the fixed method had a higher score (ICC :0.81; 95\% CI [0.17-0.96]) in comparison to the non-fixed method (ICC: 0.91 (95\% CI [0.54-0.98]) (Figure 2).

\section{DISCUSSION:}

This study demonstrated that the evaluation of the upper limb muscle strength with an isometric dynamometer showed almost perfect reliability as well as reproducibility. In the study by Saccol et al. ${ }^{21}$, reproducibility of HHD for the internal and external shoulder rotators was evaluated in 20 volunteers placed in supine position using a manual method and in sedestation position using a fixed method, with a rigid device. The ICC values of the non-fixed method determined in this current study, were higher than those determined by Saccol et al. ${ }^{22}$.

In a study with 12 volunteers, Awatani et al. ${ }^{24}$ measured the reproducibility and reliability of HHD with a non-fixed method for the internal and external shoulder rotators. The intra-examiner ICC value (ICC: 0.94, 95\% CI [0.81-0.98]) for the measurement of shoulder internal rotator strength determined by Awatani et al. was lower than the ICC value of 0.96 determined in this current study. On the contrary, the inter-examiner ICC value (0.96 [95\% CI: 0.87-0.99]) was higher than the value of 0.93 found in this study.

Dowman et al. ${ }^{25}$ evaluated the reproducibility of HHD with a non-fixed method for the measurement of elbow flexor and knee extensor strength in 30 patients with interstitial lung disease. The evaluation protocol for the elbow flexors was similar to that of this study. As a result, the reproducibility ICC values were similar to those of this study, ICC $0.98,95 \%$ CI [0.96-0.99] and ICC of 0.97 [0.93-0.99], respectively. This supports the notion that the reproducibility of HHD for evaluation of elbow strength in diverse populations remains high.

This is the first study to evaluate the reproducibility and reliability for ten different upper limb movements, thus demonstrating the clinical importance of this publication. Study limitations include: the absence of non-dominant limb measurements, which is justified by the extension of the evaluation protocol to the ten main upper limb muscle groups; as well as the absence of measurements such as pronation and supination of the elbow, and radial and ulnar deviation of the wrist.

The reproducibility of the evaluation of the upper limb muscle strength with HHD was very good, as was the reliability. The comparison of the fixed and non-fixed method of HHD assessment demonstrated the superior reproducibility and reliability of the non-fixed method in comparison to the fixed method. Only HHD for elbow extensors and external shoulder rotators showed equivalence between the evaluation methods.

\section{ABBREVIATIONS:}

ANOVA: Analysis of Variance

CI: Confidence Interval

HHD: Hand-Held Dynamometry

ICC: Intraclass Correlation Coefficient

ROM: Range of Motion 


\section{SD: Standard Deviation}

\section{REFERENCES:}

1. Cruz-Jentoft AJ, Bahat G, Bauer J, Boirie Y, Bruyére O, Cederholm T et al. Sarcopenia: revised European consensus on definition and diagnosis. Age and Ageing 2018; 0: 1-16. doi: 10.1093/ageing/afy169

2. Fontes AP, Fernandes AA, Botelho MA. Funcionalidade e incapacidade: aspectos conceptuais, estruturais e de aplicação da Classificação Internacional de Funcionalidade, Incapacidade e Saúde (CIF). Rev Port Saúde Pública. 2010;28(2):171-8.

3. Porto JM, Nakaishi APM, Cangussu-Oliveira LM, Freire RC, Spilla SB, de Abreu DCC, Relationship between grip strength and global muscle strength in community-dwelling older people, Archives of Gerontology and Geriatrics (2019), https://doi.org/10.1016/j.archger.2019.03.005

4. Kemmler W, von Stengel S, Schoene D. Longitudinal Changes in Muscle Mass and Function in Older Men at Increased Risk for Sarcopenia - The FrOST-Study. J Frailty Aging. 2019;8(2):57-61. doi: 10.14283/jfa.2019.9.

5. Schrama PP, Stenneberg MS, Lucas C, van Trijffel E. Intraexaminer reliability of hand-held dynamometry in the upper extremity: a systematic review. Arch Phys Med Rehabil. 2014 Dec;95(12):2444-69. doi: 10.1016/j.apmr.2014.05.019.

6. Polat S, Öğüt E, Göker P, Bozkır MG, Yücel AH. Reference values for hand muscle strength evaluation methods in healthy young adults. Journal of Back and Musculoskeletal Rehabilitation. 2019;1-9. doi:10.3233/bmr-170915

7. Meyer C, Corten K, Wesseling M, Peers K, Simon J-P, et al. Test-Retest Reliability of Innovated Strength Tests for Hip Muscles. PLoS ONE 2013;8(11): e81149. doi:10.1371/journal.pone.0081149

8. Edwards RHT and McDonnell M. Hand-held dynamometer for evaluating voluntary muscle function. The Lancet 1974; 757-8

9. Lohmann Siegel K, Hicks JE, Koziol DR, Gerber LH, Rider LG. Walking ability and its relationship to lower-extremity muscle strength in children with idiopathic inflammatory myopathies. Arch Phys Med Rehabil. 2004;85:767-771

10. Merlini L, Bertini E, Minetti C, et al. Motor function-muscle strength relationship in spinal muscular atrophy. Muscle Nerve.2004;29:548- 552

11. Mentiplay BF, Perraton LG, Bower KJ, Adair B, Pua Y-H, Williams GP, et al. Assessment of Lower Limb Muscle Strength and Power Using Hand-Held and Fixed Dynamometry: A Reliability and Validity Study. PLoS ONE 2015; 10(10):e0140822. doi:10.1371/journal.pone.0140822

12. Martin HJ, Yule V, Sydall HE, Dennison EM, Cooper C, Aihie Sayer A. Is Hand- Held Dynamometry Useful for the Measurement of Quadriceps Strength in Older People? A Comparison with the Gold Standard Biodex Dynamometry. Gerontology 2006;52:154-159 doi: 10.1159/000091824

13. Clavet H, Hébert PC, Fergusson D, Doucette S, Trudel G. Joint contracture following prolonged stay in the intensive care unit. CMAJ. 2008;178(6):691-7.

14. Fieseler G, Molitor T, Irlenbusch L, Delank KS, Laudner KG, Hermassi S et al. Intrarater reliability of goniometry and hand held dynamometry for shoulder and elbow examinations in female team handball athl etes and asymptomatic volunteers. Arch Orthop Trauma Surg. 2015;135(12):1719-26. doi:10.1007/s00402-015-2331-6

15. Davis PR, Mckay MJ, Baldwin JN, Burns J, Pareyson D, Rose KJ. Repeatability, consistency, and acurracy of hand-held dynamometry with and without fication fot measuring ankle plantarflexion strength in healthy adolescentes and adults. Muscle Nerve. 2017;56:896-900. doi:10.1002/mus25576

16. Almeida GPL, Albano TR, Melo AKP. Hand-held dynamometer identifies asymmetries in torque of the quadriceps muscle after anterior cruciate ligament reconstruction. Knee Surg Sports Traumatol Arthrosc. 2018 Oct 30. doi: 10.1007/s00167-018-5245-3. [Epub ahead of print]

17. Hébert LJ, Maltais DB, Lepage C, Saulnier J, Crête M. Hand- Held Dynamometry Isometric Torque Reference Values for Childrenand Adolescents. Pediatr Phys Ther. 2015 Winter;27(4):414-23. doi: 10.1097/PEP.0000000000000179

18. Van der Ploeg, Fidler V, Oosterhuis HJ. Hand-held myometry: reference values. J Neurol Neurosurg 
Psychiatry. 1991;54(3):244-7

19. Genlin L, Jianjun L, Hongjun Z, Ying Z, Chunxia H, Ying Z et al. A simple way to improve the relative and absolute reliability of handheld dynamometer measurements using learners. Int J Clin Exp Med. 2016;9(1):199-208

20. Rheault W, Beal JL, Kubik KR, Nowak TA, Shepley JA. Intertester reliability of the Hand-held dynamometer for wrist flexion and extension. Arch Phys Med Rehabil. 1989 Dec;70(13):907-10.

21. Cadogan A, Laslett M, Hing W, McNair P, Williams M. Reliability of a new hand-held dynamometer in measuring shoulder range of motion and strength. Manual Therapy. 2011: 16 97e101. doi:10.1016/j.math.2010.05.005

22. Saccol MG, Dos Santos G, Oliano HJ. Inter- and intra-examiner reliability of the strength of shoulder rotators in different positions using isometric dynamometry. Fisioter Pesqui. 2017;24(4):406-11. doi: 10.1590/1809-2950/17257624042017

23. Weir JP. Quantifying test-retest reliability using the intraclass correlation coefficient and the SEM. J Strength Cond Res. 2005;19(1):231-40. doi: 10.1519/15184.1

24. Awatani T, Morikita I, Shinohara J, Mori S, Narial M, Tatsumi Y et al. Intra- and inter-rater reliability of isometric shoulder extensor and internal rotator strength measurements performed using a hand-held dynamometer. J. Phys. Ther. Sci. 2016;28: 3054-9

25. Dowman L, McDonald CF, Hill CJ, Lee A, Barker K, Boote C et al. Reliability of the hand held dynamometer in measuring muscle strength in people with interstitial lung disease. Physiotherapy. 2016;102(3):249-55. doi: 10.1016/j.physio.2015.10.002.

26. von Haehling S, Morley JE, Coats AJS, Anker SD. Diretrizes éticas para publicação no Journal of Cachexia, Sarcopenia and Muscle: atualização 2017. J Cachexia Sarcopenia Muscle 2017; 8: 1081-1083

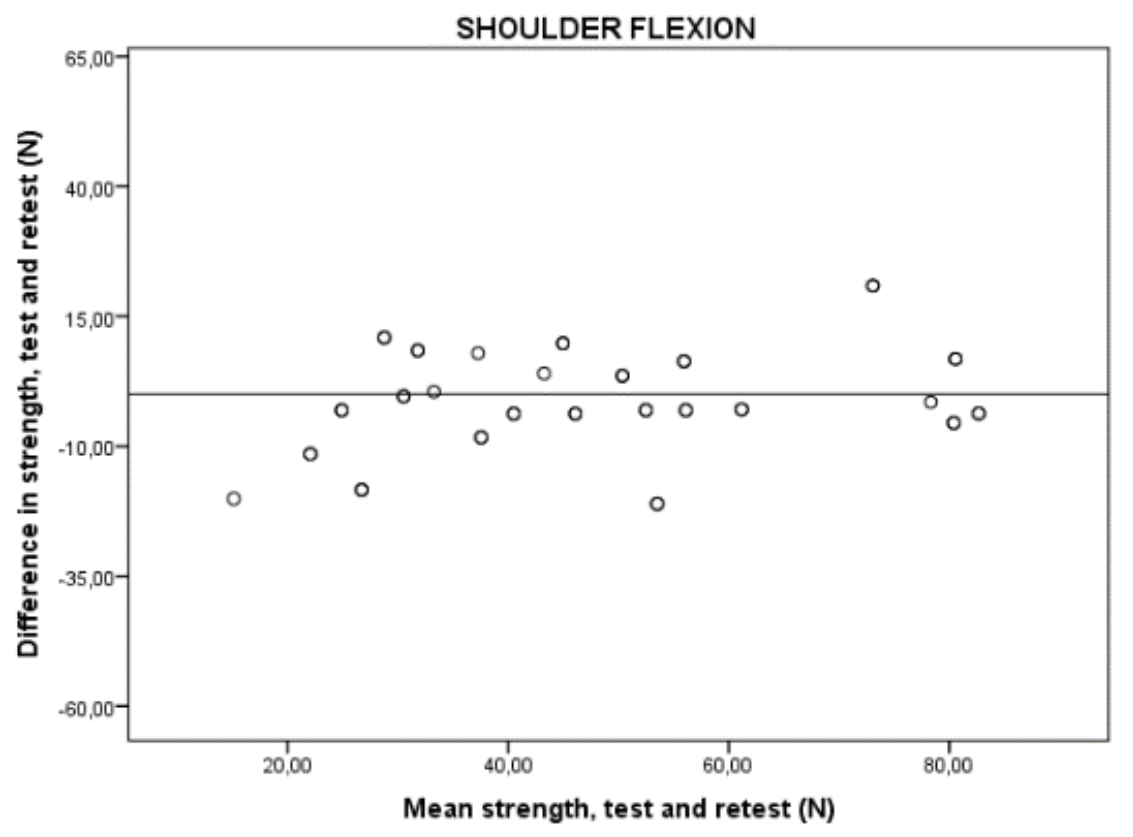


SHOULDER FLEXION
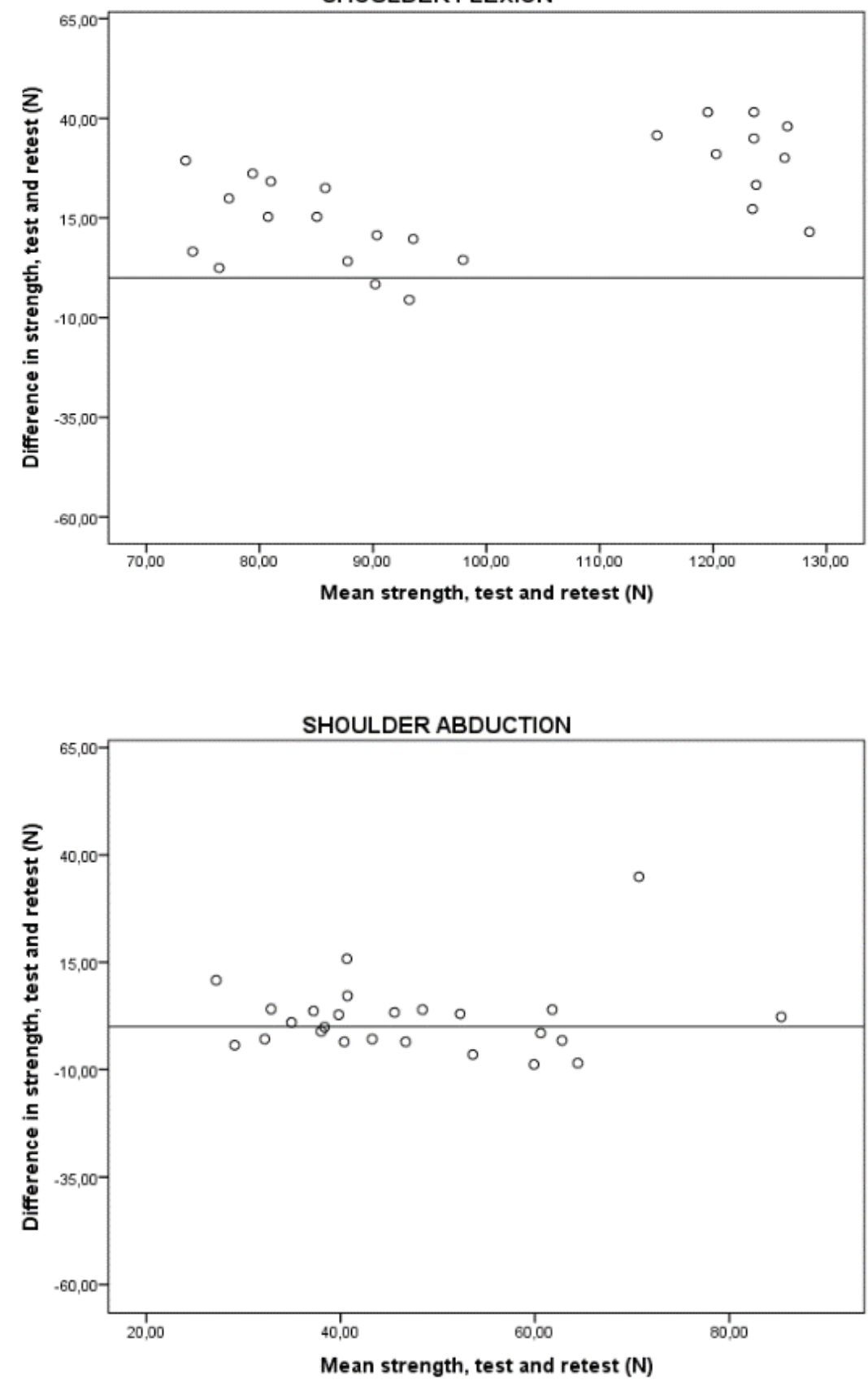

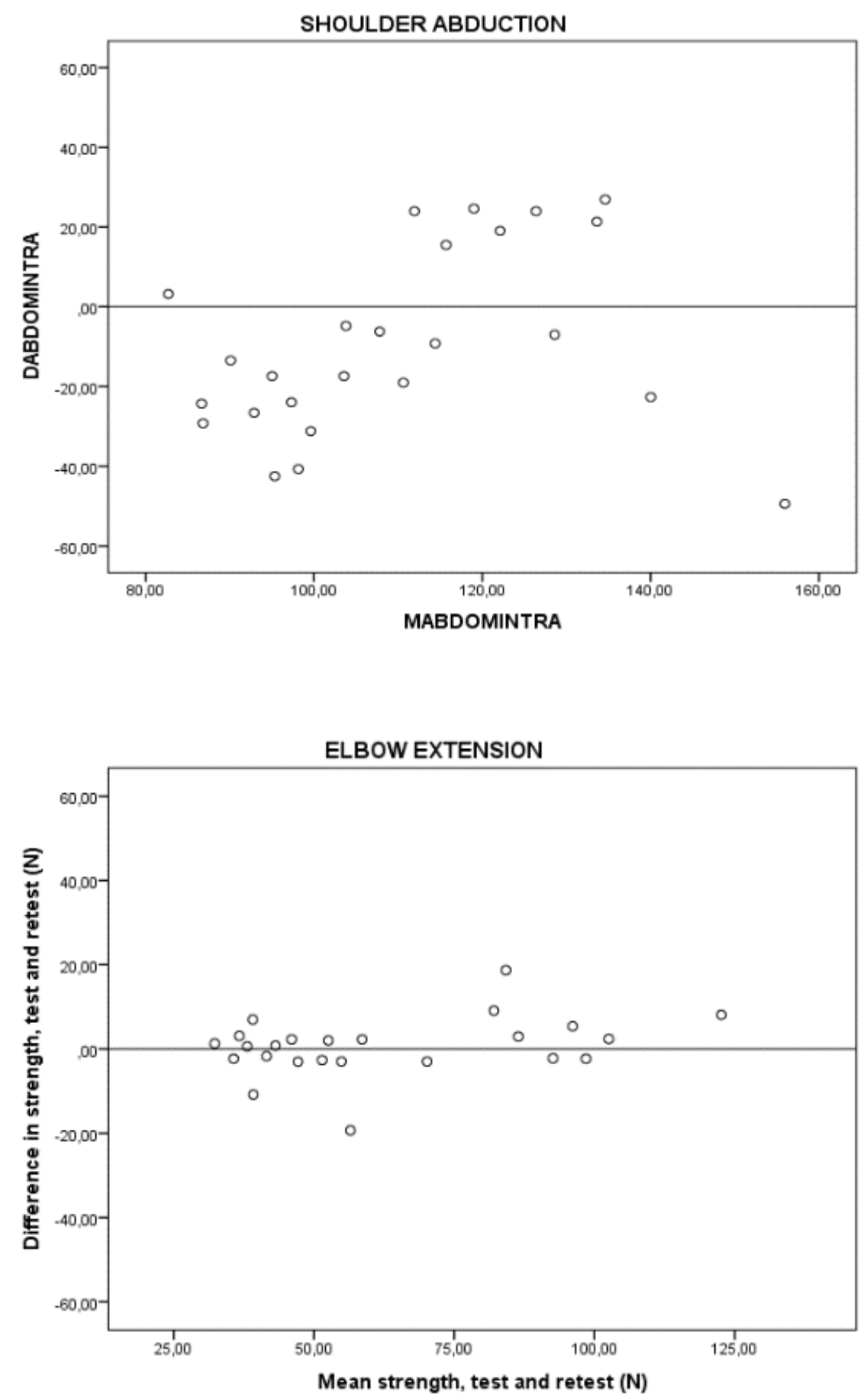

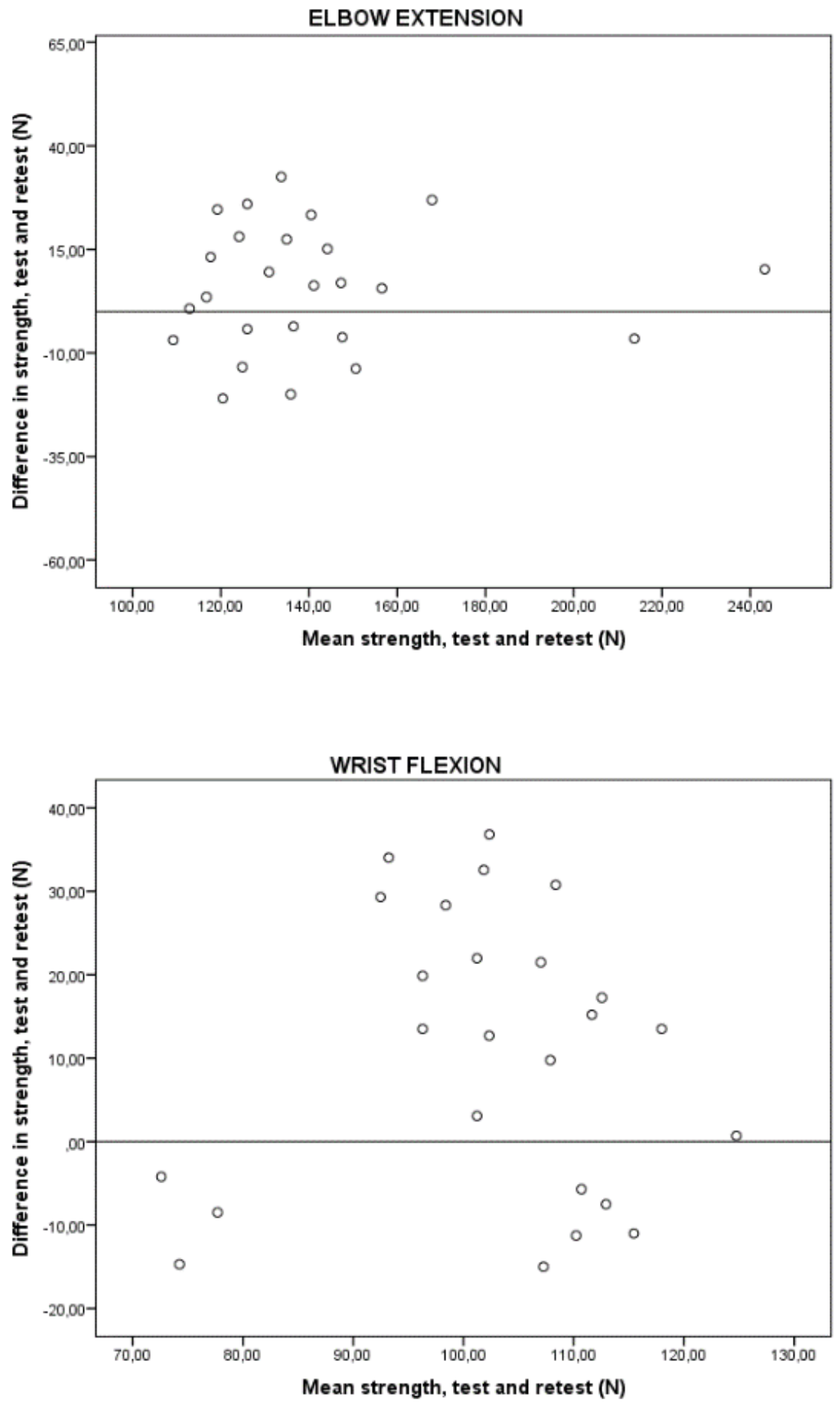


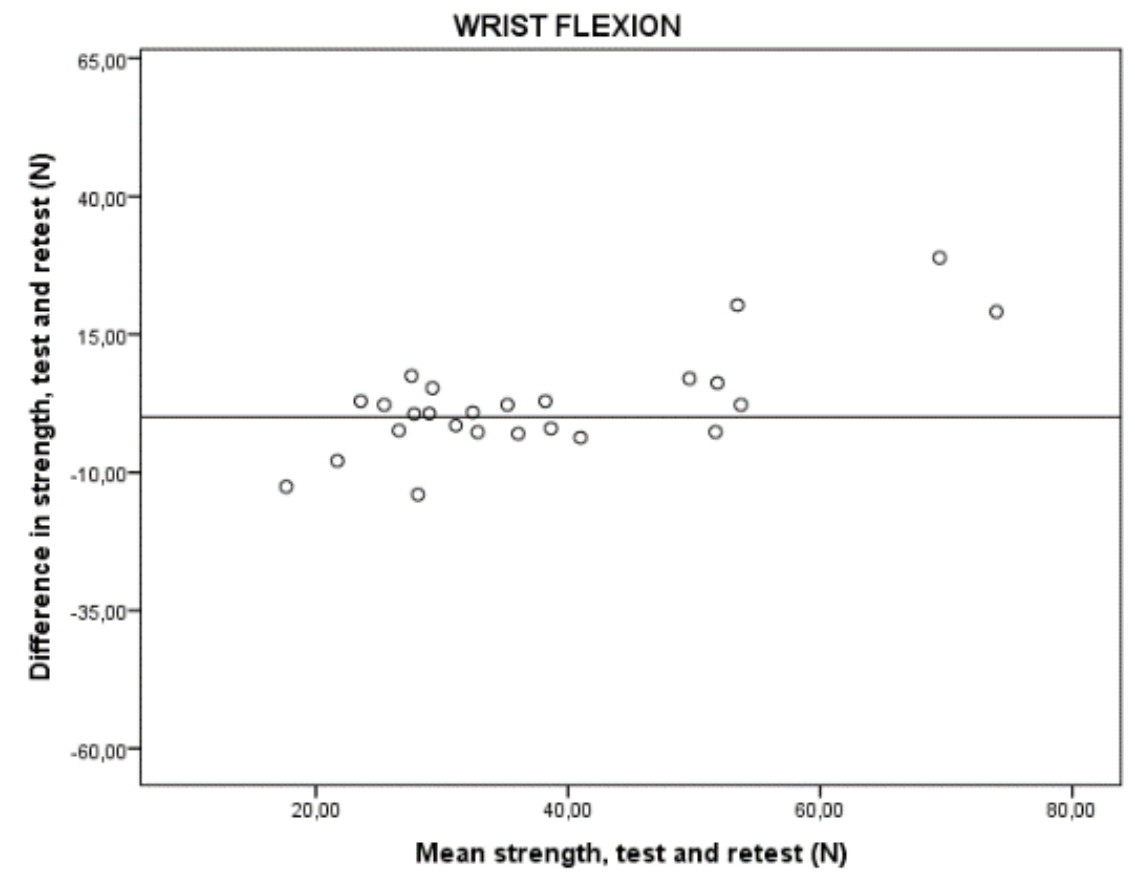

Figure 01: The Bland Altmann plots provide the agreement between non-fixed HHD (A) and fixed HHD (B).

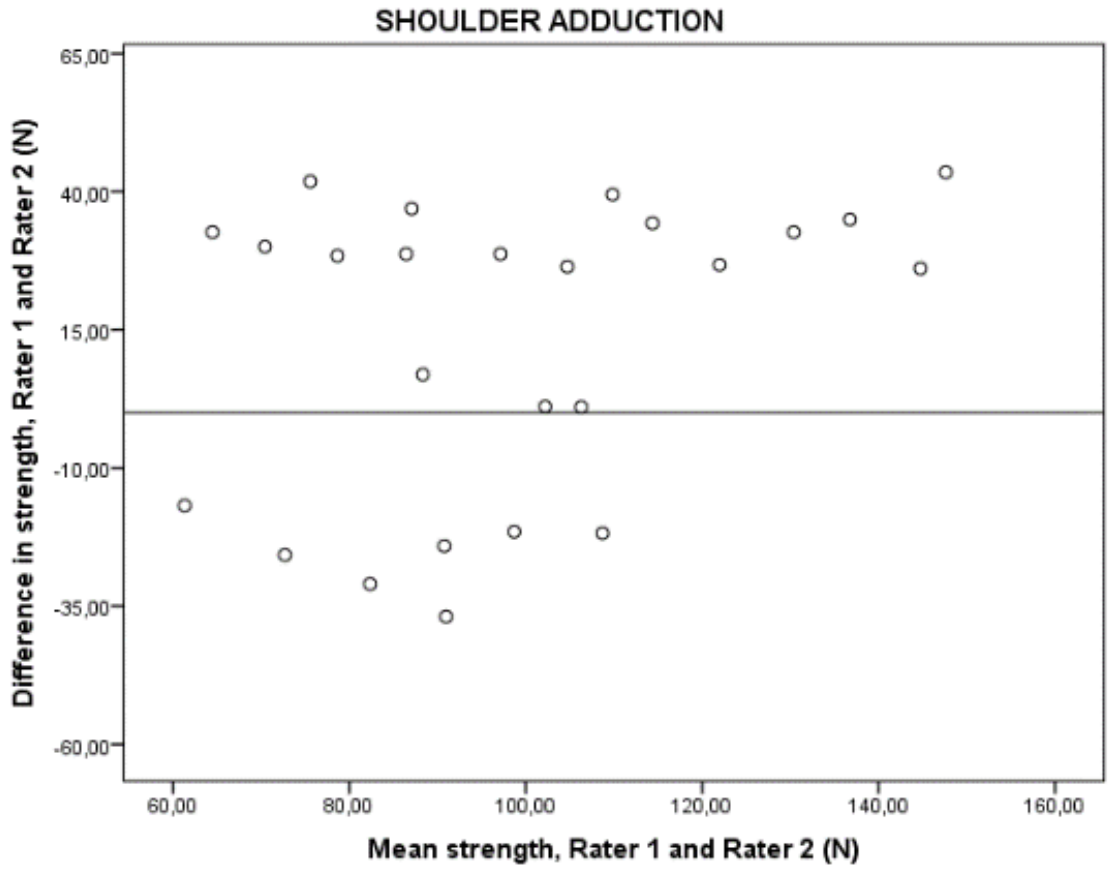



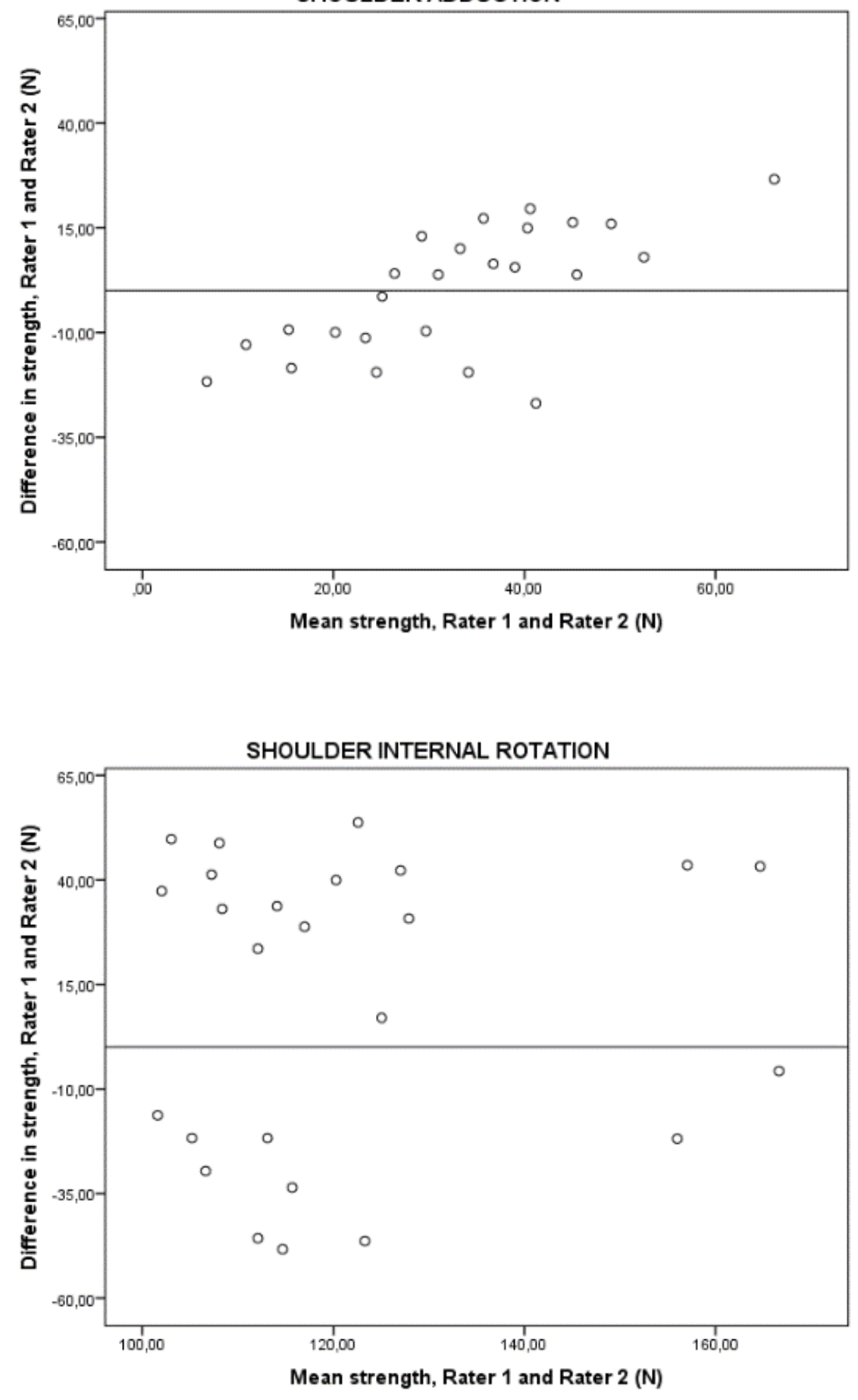

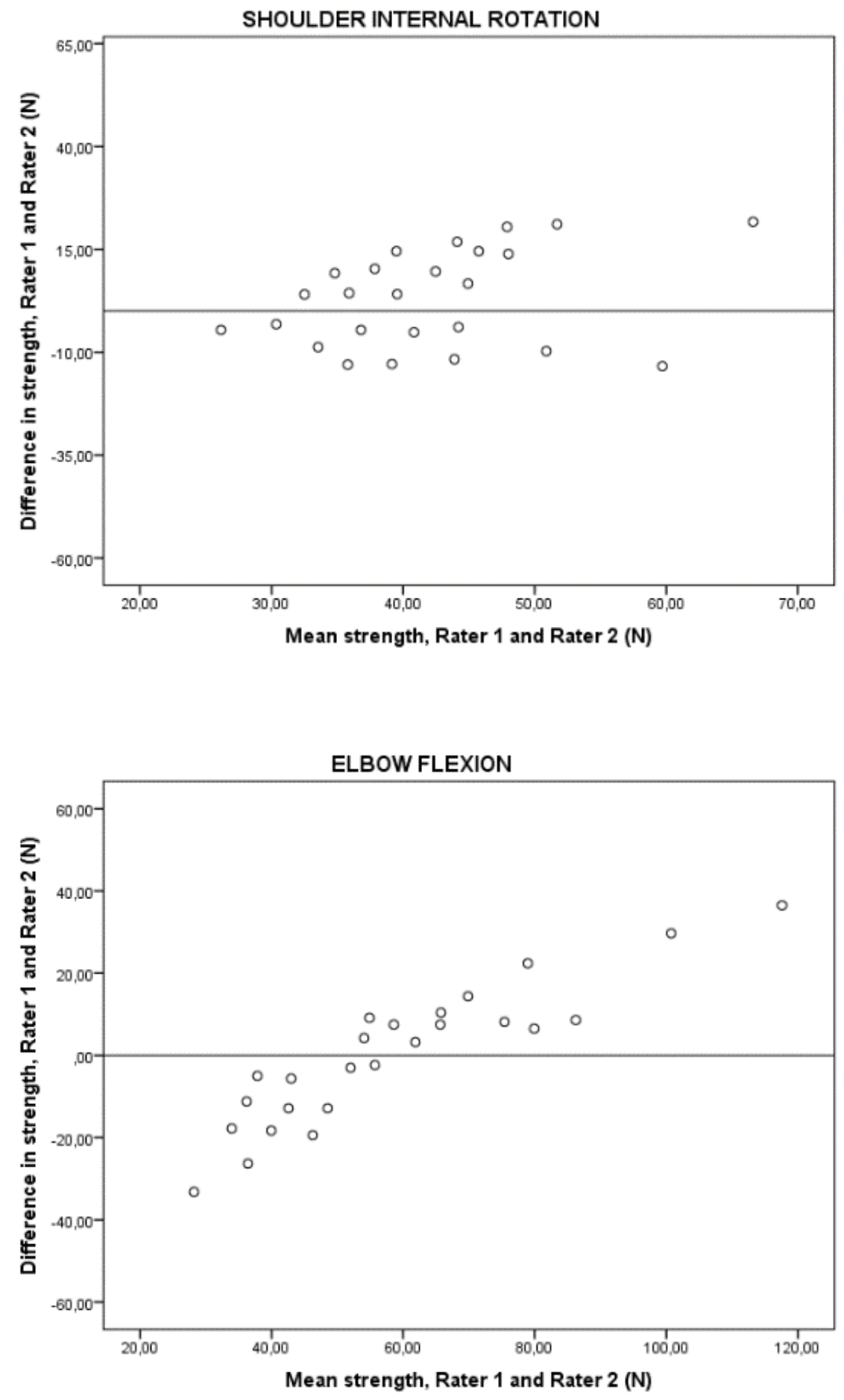

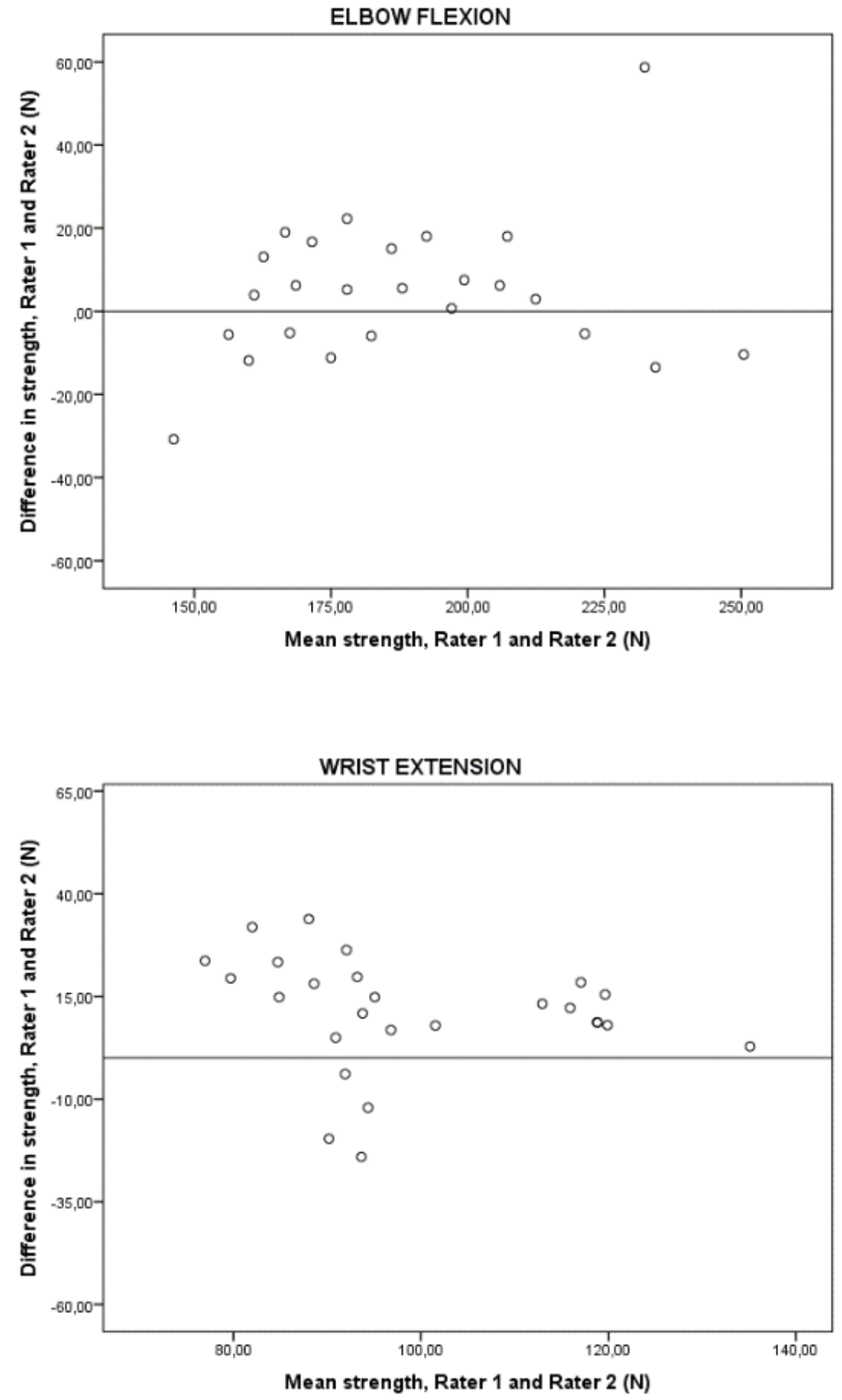


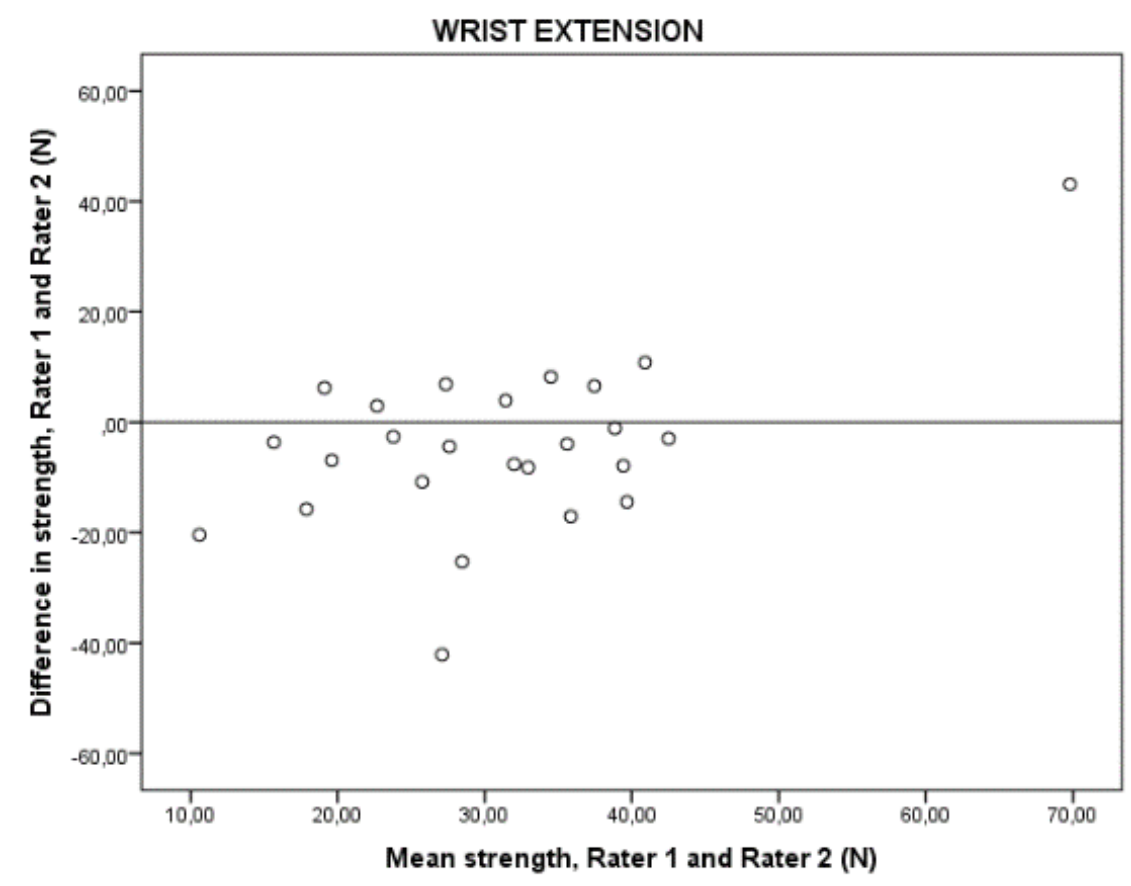

Figure 02: Reliability in The Bland Altmann plots provide the agreement between non-fixed HHD (A) and fixed HHD (B).

\section{Hosted file}

Table 01.docx available at https://authorea.com/users/291816/articles/419317-comparison-offixed-and-non-fixed-methods-of-hand-held-dynamometry

\section{Hosted file}

Table 02.docx available at https://authorea.com/users/291816/articles/419317-comparison-offixed-and-non-fixed-methods-of-hand-held-dynamometry

\section{Hosted file}

Table 03.docx available at https://authorea.com/users/291816/articles/419317-comparison-offixed-and-non-fixed-methods-of-hand-held-dynamometry

\section{Hosted file}

Table 4.docx available at https://authorea.com/users/291816/articles/419317-comparison-offixed-and-non-fixed-methods-of-hand-held-dynamometry

\section{Hosted file}

Table 05.docx available at https://authorea.com/users/291816/articles/419317-comparison-offixed-and-non-fixed-methods-of-hand-held-dynamometry 